\title{
VIOLENCIA ESCOLAR Y CONSUMO DE SUSTANCIAS EN ESTUDIANTES MEXICANOS DE SECUNDARIA do
}

\author{
Julio César Vázquez-Colunga ${ }^{a}{ }^{\circledR}$, Mario Ángel-González ${ }^{\circledR}{ }^{\circledR}$, Cecilia \\ Colunga-Rodríguez a \& ${ }^{b}{ }^{\circ}$, Bertha Alicia Colunga-Rodríguez ${ }^{c} \mathbb{D}$, \\ Francisco Javier Pedroza-Cabrera ${ }^{\mathrm{d}} \mathbb{O}, \&$ Claudia Liliana Vázquez- \\ Juárez ${ }^{\text {a1 (i) }}{ }^{2}$
}

\author{
Universidad de Guadalajara, Guadalajara, México a, Instituto Mexicano \\ del Seguro Social, Guadalajara Jalisco, México ${ }^{\text {b }}$; Escuela Normal \\ Superior de Jalisco, Guadalajara, México ${ }^{\text {; }}$ Universidad Autónoma de \\ Aguascalientes, Aguascalientes, México ${ }^{\mathrm{d}}$.
}

\section{RESUMEN}

Se realizó un estudio transversal con muestra de 2211 adolescentes de cuatro escuelas públicas. Se aplicó escala de seguridad en la escuela y consumo de sustancias psicoactivas. La edad fue 13.6 años. Las peleas físicas en propiedad escolar fue la conducta violenta más reportada, $44.1 \%$ refieren participación en al menos una pelea en el año escolar. Para violencia escolar se encontraron diferencias en cuanto a género, grado escolar, calificaciones y turno escolar $(p<.05)$. En consumo de sustancias, $33.6 \%$ refirió beber un vaso de alcohol en el último mes, $14.3 \%$ fumó cigarros de tabaco y el $9.1 \%$ de marihuana. En consumo de sustancias, sólo hubo diferencias por grado, calificaciones y turno escolar $(p<.05)$, no entre sexo. Entre 3 y 4 de cada 10 adolescentes, refieren conductas de riesgo, los resultados sugieren prevención priorizando grupos vulnerables, en particular hombres de mayor grado escolar, turno vespertino y menores calificaciones.

\section{Palabras Claves}

violencia escolar; consumo de sustancias; escuela secundaria; adolescencia

\begin{abstract}
A cross-sectional study conducted, involving 2211 adolescents from four public schools. A scale of school safety and psychoactive substance use was applied. The age was 13.6 years. Physical fights on school property were the most reported violent behavior, $44.1 \%$ participation in at least one fight in the school year. For school violence, there are many differences in terms of gender, grade, scores and school shift. In substance consumption, 33.6\% reported having drunk a glass of alcohol in the last month, 14.3\% reported having smoked tobacco cigars and $9.1 \%$ of marijuana $(\mathrm{p}<.05)$. In substance use, there were only differences by grade, grades and school shift ( $\mathrm{p}<.05)$, not between sexes. Approximately 3 and 4 out of 10 adolescents, report risk behaviors, the results suggest prevention, prioritizing vulnerable groups, particularly men with a higher school grade, afternoon shift and low school grades.
\end{abstract}

Keywords

school violence; substance use; middle school; adolescence

\footnotetext{
1 Correspondence about this article should be addressed to Claudia Liliana Vázquez-Juárez: claudia.vazquezjuarez@ensj.edu.mx

2 Conflicts of Interest: The authors declare that the research was conducted in the absence of any commercial or financial relationships that could be construed as a potential conflict of interest.
} 


\section{SCHOOL VIOLENCE AND SUBSTANCE USE IN MIDDLE SCHOOL MEXICAN STUDENTS}

\section{Introducción}

La secundaria es una etapa de adaptación entre el adolescente y su medio, cuando este proceso no es exitoso, pueden generarse dificultades en el desarrollo. Estas problemáticas pueden manifestarse en indicadores como poco apego y abandono escolar, aparición de adicciones y otras alteraciones de salud mental (Iachini et al., 2013). La violencia escolar y el consumo de sustancias psicoactivas (SPA) son problemas relevantes que surgen durante esta etapa, su impacto deletéreo se refleja en las consecuencias que generan para el adolescente y para el contexto donde se encuentra inserto (American Academy of Child and Adolescent Psychiatry, 2015).

La violencia escolar es un subtipo de comportamiento agresivo, cuyo objetivo intencional es causar daño físico, emocional y/o social a otros dentro del espacio escolar (Yarnell et al., 2014). Algunas conductas que se califican como violencia escolar son la agresión verbal, bullying, peleas físicas, robo, daño de propiedad, etc. (Algozzine \& McGee, 2011). La exposición a la violencia durante la adolescencia está clasificada como un problema de salud pública a nivel internacional, debido a los importantes efectos académicos, psicológicos, conductuales y sociales que provoca, no sólo en los participantes directos, sino en toda la comunidad educativa (Hutchinson, 2012; Milam et al., 2010).

En América Latina, se realizó un estudio que incluyó a 16 países y se encontró que el $51.1 \%$ de los estudiantes de sexto año de primaria, reportaron haber sufrido algún tipo de violencia escolar, siendo las agresiones más frecuentes el robo en un 39.4\%, la violencia verbal en un $26.6 \%$ y la violencia física en el $16.5 \%$ de los participantes (Román \& Murillo, 2011). Por su parte, en estudio realizado en México, se reportó que la agresión más común en la escuela es el robo con incidencia de 43.6\%, seguida de agresiones físicas con $14.5 \%$ y agresiones verbales (burlas) con $13.6 \%$ (Aguilera et al., 2007).

Como fenómeno asociado a la violencia escolar en adolescentes, se encuentra el consumo de sustancias psicoactivas (SPA). La Organización Mundial de la Salud (2004), lo define como el uso experimental, moderado o frecuente de alguna droga, entendida como aquella sustancia que provoca alguna alteración en el estado de ánimo y que puede llegar a producir dependencia física o psicológica. Durante la adolescencia, especialmente en la etapa de la secundaria, es común encontrar distintos tipos de patrones de consumo 
de SPA entre los estudiantes, siendo el consumo experimental uno de los más frecuentes (Aguilera, et al., 2007).

Las dos principales sustancias con las que el adolescente suele tener contacto durante la secundaria son alcohol y tabaco, debido principalmente a su disponibilidad y aceptación social (Meneses et al., 2013). Los resultados de un estudio en México, indican que $24.6 \%$ de los estudiantes de secundaria reportaron haber consumido alcohol en distintas cantidades, el 15\% de ellos consumió uno o dos vasos durante el año escolar. Por otra parte, $12 \%$ mencionan haber fumado uno o dos cigarros en un día (Aguilera, et al., 2007).

En la Encuesta Nacional de Consumo de Drogas, Alcohol y Tabaco del año 2017 (ENCODAT), se reportó una prevalencia del 3.1\% en el consumo de sustancias ilegales en población adolescente de 12 a 17 años de edad (ENCODAT, 2017). Por su lado en la Encuesta Nacional de Consumo de Drogas en Estudiantes (ENCODE) realizada en el 2014, se reportó que el porcentaje de estudiantes de secundaria y bachillerato que alguna vez consumieron cualquier droga, fue de $17.2 \%$ en total. Además, se encontró que la mariguana fue la droga de mayor consumo, con un $12.9 \%$ en hombres y un $8.4 \%$ en mujeres; seguida por los inhalables, con un 5.9\% en hombres y un 5.8\% en mujeres; la cocaína, con un $4.2 \%$ en hombres y un $2.5 \%$ en mujeres; finalmente están los tranquilizantes, con un 3.2\% en hombres y un $4.3 \%$ en mujeres (ENCODE, 2015). El consumo de sustancias durante la adolescencia, ya sean lícitas o ilícitas, es un problema serio, a corto y largo plazo debido a que sus efectos pueden perduran hasta la vida adulta.

A pesar de la relevancia mundial de la violencia escolar y del consumo de SPA, en nuestro país se han realizado pocas investigaciones desde la perspectiva de la salud pública, por lo que es necesario recabar información actualizada que permita el futuro desarrollo de estrategias de prevención desde los entornos escolares y con componentes basados en la investigación (Valadez et al., 2018). Debido a lo anterior, el objetivo de esta investigación fue describir la prevalencia de conductas de violencia escolar y consumo de SPA en estudiantes de secundaria de Guadalajara, así como las diferencias observadas en cuanto al género y algunas variables escolares.

\section{Método}




\section{Diseño}

Estudio de tipo comparativo, no experimental y de corte transversal.

\section{Participante}

Estudiantes de cuatro escuelas secundarias públicas en Guadalajara. Las escuelas fueron seleccionadas aleatoriamente, incluyendo a la totalidad de los estudiantes que se encontraban presentes al momento de la aplicación del instrumento.

La muestra final incluyó 2211 estudiantes, con una media de edad de 13.6 años. De los participantes el $51 \%$ se identificaron con el género femenino y el $49 \%$ con el género masculino. Con respecto al grado escolar, $40.08 \%$ de los participantes cursaban el primer grado, $34.14 \%$ el segundo grado y el $25.78 \%$ el tercer grado. Por otro lado el 54 $\%$ de los estudiantes asistían al turno matutino y el $46 \%$ al turno vespertino. Acerca de las calificaciones, el promedio reportado en la última boleta fue de 8.26.

\section{Instrumentos}

Las escalas utilizadas en esta investigación fueron adaptadas del MDS3 School Climate (Bradshaw et al., 2014), instrumento utilizado para evaluar las variables correspondientes al clima escolar y adaptado a población mexicana por (Orozco, 2017). Este instrumento se divide en tres escalas: seguridad, compromiso escolar y entorno escolar. Para esta investigación se utilizó la escala seguridad, la cual obtuvo un Alfa de Cronbach de 0.81. Así mismo, la escala de seguridad tiene dos subescalas: seguridad en la escuela y consumo de sustancias, las cuales se describen a continuación:

Seguridad en la escuela. Es una escala de 17 preguntas divididas en tres dimensiones. Los primeros cuatro ítems abordan la percepción de la seguridad en la escuela y de las actitudes de estudiantes y maestros frente al bullying. En la segunda dimensión se incluyen siete ítems que preguntan acerca de qué tanto es un problema en la escuela la existencia de diversas conductas violentas. Ambas secciones con opciones de respuesta tipo Likert de cuatro puntos, que van de totalmente de acuerdo a totalmente en desacuerdo. Finalmente, se presentan seis ítems acerca de la participación del estudiante en conductas violentas como agresor o víctima dentro de un periodo específico, incluye ítems como durante los últimos 12 meses, ¿cuántas veces te peleaste físicamente 
dentro de la escuela?, con cinco opciones de respuesta que van de cero días a seis o más días.

Consumo de sustancias. Es una escala con 15 preguntas y cuatro dimensiones. Los primeros siete ítems tratan sobre el consumo de sustancias en los últimos 30 días, incluyendo alcohol, tabaco, marihuana, medicinas sin receta médica y otro tipo de drogas (Inciensos herbales, potpurrí, sales de baño, pegamento, solventes), tiene seis opciones de respuesta que van de cero días a 30 días. Además, se incluyen cinco ítems acerca de la facilidad de acceso percibido a las sustancias mencionadas anteriormente, con opciones de respuesta en escala tipo Likert de cuatro puntos. Por último, se incluyen tres ítems acerca del riesgo para la salud que perciben en el consumo cotidiano de alcohol, cigarros y marihuana, con opciones de respuesta en escala tipo Likert de cuatro puntos que van de ningún riesgo a gran riesgo.

\section{Procedimento}

Se llevaron a cabo reuniones con los directores de las secundarias seleccionadas y se les explicaron los objetivos y procedimientos necesarios para el desarrollo de la investigación. Los directores aceptaron que se llevara a cabo la investigación en sus planteles escolares. Después se envió el consentimiento informado a los padres de familia o tutores de los estudiantes de cada escuela, para que lo firmaran. Una semana después se comenzó la aplicación de los instrumentos, el cual fue contestado en línea y en una sola sesión llevada a cabo dentro de los salones de cómputo. Al inicio de cada aplicación se leyó la información relacionada con las consideraciones éticas de la investigación y se tomó el asentimiento verbal de los estudiantes para participar libremente; se leyeron las instrucciones de respuesta del instrumento y se procedió a iniciar la aplicación colectiva, con respuesta individual por parte de los estudiantes. Al finalizar de contestar el cuestionario los participantes regresaban a su salón de clases.

\section{Análisis de datos}

Los datos recabados se analizaron con el programa SPSS versión 20.0. Se obtuvieron estadísticos descriptivos. También se realizaron pruebas de diferencias con la $\mathrm{t}$ de Student para grupos independientes y ANOVA de un factor. Se consideraron como significativo los valores de $p<.05$. 


\section{Consideraciones éticas}

Esta investigación se consideró de riesgo mínimo ya que sólo se utilizaron variables de índole psicológica. Antes de ser incluido en la investigación, cada participante y su padre otorgaron el consentimiento informado. Se enfatizó en la confidencialidad de la información y se explicó que la participación era voluntaria y sin remuneración económica o académica. El estudio fue aprobado y autorizado por el Comité de Ética de la Normal Superior de Jalisco, adscrita a la Secretaria de Educación Pública.

\section{Resultados}

\section{Violencia escolar}

En la tabla 1 se observa que el $90.4 \%$ de los participantes refieren sentirse seguros en la escuela, mientras que cerca del $88.1 \%$ mencionan sentirse seguros yendo y viniendo de la escuela. También se puede observar que el $80.6 \%$ de los participantes considera que los adultos tratan de detener el bullying, por otro lado el $54.4 \%$ considera que los estudiantes tratan de detener el bullying.

Tabla 1.

Frecuencias y porcentajes de percepción de la seguridad en la escuela

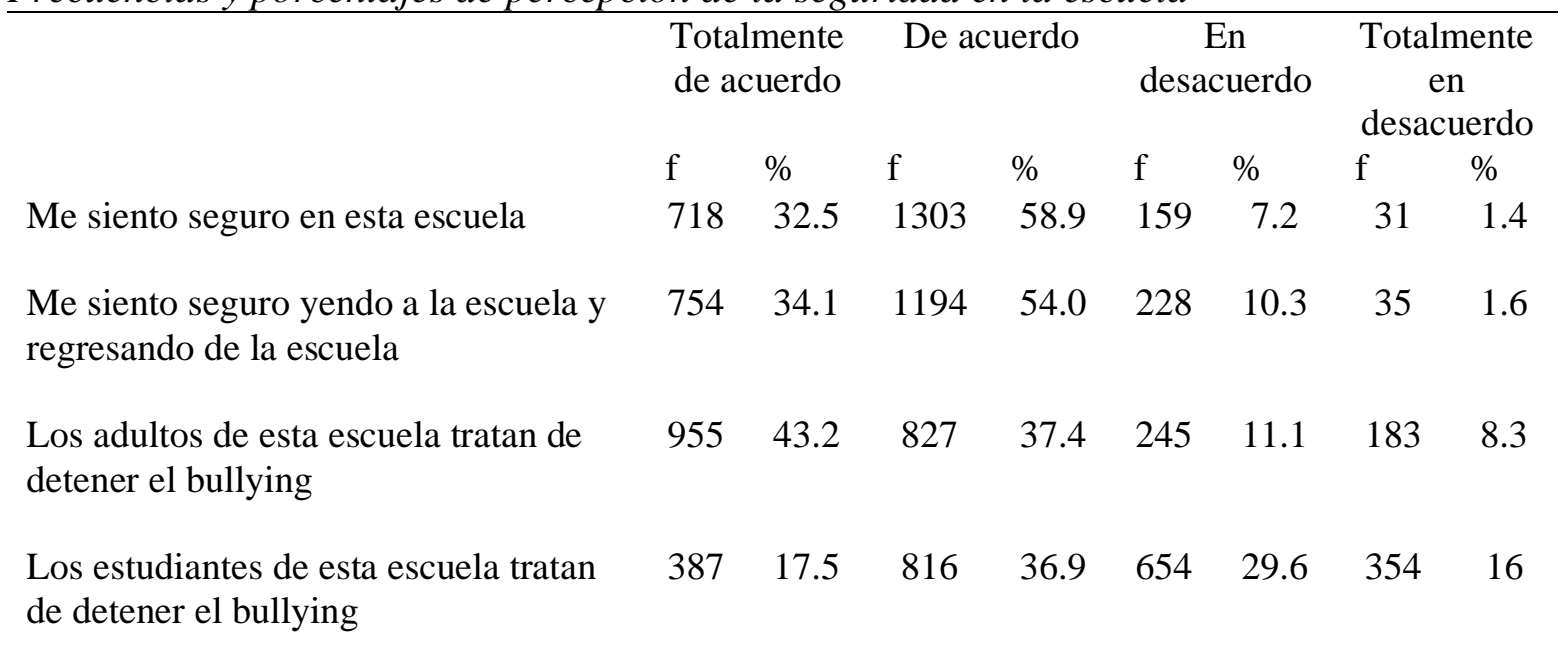

Fuente: elaboración propia $\mathrm{f}=$ frecuencia $\%=$ porcentaje $\mathrm{N}=2211$

Asimismo, se analizó la participación de los estudiantes en situaciones de violencia escolar, ya sea como víctimas, agresores u observadores. Los resultados se presentan en la tabla 2, donde se observa que las peleas físicas son una de las conductas violentas en la que participan con mayor frecuencia los estudiantes. 
Tabla 2.

Frecuencias y porcentajes de las conductas violentas dentro de la escuela durante los últimos 30 días

\begin{tabular}{|c|c|c|c|c|c|c|c|c|c|c|}
\hline & \multicolumn{10}{|c|}{ Frecuencia de desarrollo de la conducta } \\
\hline & \multicolumn{2}{|c|}{0 días } & \multicolumn{2}{|c|}{1 día } & \multicolumn{2}{|c|}{2 o 3 días } & \multicolumn{2}{|c|}{4 o 5 días } & $\begin{array}{c}6 \text { o } \\
\text { días } \\
\mathrm{f}\end{array}$ & más \\
\hline $\begin{array}{l}\text { Portar armas, cuchillo o } \\
\text { pistola, dentro de } \\
\text { propiedad escolar }\end{array}$ & $\begin{array}{c}\mathrm{f} \\
2116\end{array}$ & $\begin{array}{c}\% \\
95.7\end{array}$ & $\begin{array}{c}\mathrm{f} \\
64\end{array}$ & $\begin{array}{c}\% \\
2.9\end{array}$ & $\begin{array}{c}\mathrm{f} \\
20\end{array}$ & $\begin{array}{c}\% \\
0.9\end{array}$ & $\begin{array}{l}\mathrm{f} \\
2\end{array}$ & $\begin{array}{c}\% \\
0.1\end{array}$ & $\begin{array}{l}\mathrm{f} \\
9\end{array}$ & $\begin{array}{c}\% \\
0.4\end{array}$ \\
\hline Participar en peleas físicas & 1457 & 65.9 & 351 & 15.9 & 247 & 11.1 & 68 & 3.1 & 88 & 4.0 \\
\hline $\begin{array}{l}\text { Ser amenazado o lastimado } \\
\text { con un arma en propiedad } \\
\text { escolar }\end{array}$ & 1977 & 89.4 & 122 & 5.5 & 62 & 2.8 & 16 & 0.7 & 34 & 1.5 \\
\hline
\end{tabular}

Fuente: elaboración propia $\mathrm{f}=$ frecuencia $\%=$ porcentaje $\mathrm{N}=2211$

En esta sección se incluye una pregunta más, relacionada con la participación en pandillas delictivas y se observó que 6.9\% (152) de los participantes, refiere haber formado parte de una pandilla durante el último año escolar.

\section{Diferencias en violencia escolar en cuanto a género y algunas variables escolares}

En el caso del género, se observan diferencias estadísticamente significativas en las conductas como el portar armas $(\mathrm{t}=3.38, p<.005)$, participar en peleas físicas $(\mathrm{t}=6.28$, $p<.005)$, ser amenazado o lastimado con un arma en propiedad escolar $(\mathrm{t}=3.6, p<.005) \mathrm{y}$ pertenecer a una pandilla $(\mathrm{t}=5.03, p<.005)$, observando que los varones puntuaron con medias mayores que las mujeres.

Con relación al turno en la escuela, se encontraron diferencias significativas en conductas relacionadas con la participación en pandillas delictivas $(\mathrm{t}=-3.63, p<.005)$. En estas conductas, los alumnos del turno vespertino obtuvieron medias mayores que los del matutino.

En la tabla tres, se presentan los resultados de las comparaciones realizadas de acuerdo a las calificaciones reportadas en el último periodo, se observan medias mayores en estudiantes que reportan calificaciones menores a ocho. 
VÁzquez-Colunga, Ángel-González, Colunga-Rodríguez, Colunga-Rodríguez, Pedroza-

CABRERA \& VÁZquEZ-JUÁREZ

Tabla 3.

Conductas relacionadas con violencia escolar de acuerdo a las calificaciones del último reporte

\begin{tabular}{|c|c|c|c|c|c|c|c|}
\hline \multirow{3}{*}{$\begin{array}{l}\text { Conducta relacionada } \\
\text { con violencia escolar }\end{array}$} & \multicolumn{7}{|c|}{ Calificaciones } \\
\hline & 6 & 7 & 8 & 9 & 10 & \multirow[b]{2}{*}{$\mathrm{F}$} & \multirow[b]{2}{*}{$p$} \\
\hline & & \multicolumn{4}{|c|}{$\mathrm{M}(\mathrm{DE})$} & & \\
\hline $\begin{array}{l}\text { Portar armas, cuchillo } \\
\text { o pistola, dentro de } \\
\text { propiedad escolar }\end{array}$ & $1.00(0.0)$ & $\begin{array}{l}1.10 \\
(.41)\end{array}$ & $1.08(.40)$ & $\begin{array}{l}1.04 \\
(0.2)\end{array}$ & $1.06(0.3)$ & 1.38 & .226 \\
\hline $\begin{array}{l}\text { Participar en peleas } \\
\text { físicas }\end{array}$ & $1.72(1.1)$ & $\begin{array}{l}1.88 \\
(1.3)\end{array}$ & $1.75(1.3)$ & $\begin{array}{l}1.61 \\
(1.2)\end{array}$ & $1.33(0.8)$ & $5.77^{*}$ & .000 \\
\hline $\begin{array}{l}\text { Ser amenazado o } \\
\text { lastimado con un arma } \\
\text { en propiedad escolar }\end{array}$ & $1.22(1.0)$ & $1.24(0.8)$ & $1.24(0.8)$ & $1.21(0.8)$ & $1.13(0.6)$ & 0.70 & .624 \\
\hline Pertenecer a pandillas & $0.22(.42)$ & $0.26(0.4)$ & $0.11(0.3)$ & $\begin{array}{l}0.11 \\
(0.3)\end{array}$ & $0.09(0.2)$ & $10.46^{*}$ & .000 \\
\hline
\end{tabular}

Fuente: elaboración propia $\mathrm{N}=2211 .{ }^{*} p<.000$

\section{Consumo de SPA}

En la tabla cuatro, se muestran las frecuencias y porcentajes del consumo de SPA en los últimos 30 días en la población en estudio. La sustancia de mayor prevalencia fue el alcohol, seguido por el cigarro, mariguana y medicamentos sin prescripción médica.

Tabla 4.

Frecuencias y porcentajes de consumo de SPA reportado por participantes Frecuencia de consumo

Sustancia

0 días $\quad 1$ a $2 \quad 3$ a $5 \quad 6$ a $9 \quad 10$ a $29 \quad 30$ días

$\begin{array}{lllllllllllll}\text { Alcohol } 1 \text { bebida } & 1468 & 66.4 & 475 & 21.5 & 122 & 5.5 & 68 & 3.1 & 66 & 3.0 & 13 & 0.6\end{array}$

$\begin{array}{lllllllllllll}\text { Alcohol más de } 5 & 1861 & 84.1 & 228 & 10.3 & 71 & 3.2 & 22 & 1.0 & 22 & 1.0 & 7 & 0.3\end{array}$

bebidas

$\begin{array}{lllllllllllll}\text { Cigarros } & 1897 & 85.7 & 193 & 8.7 & 57 & 2.6 & 20 & 0.9 & 24 & 1.1 & 20 & 0.9\end{array}$

$\begin{array}{lllllllllllll}\text { Marihuana } & 2010 & 90.9 & 104 & 4.7 & 40 & 1.8 & 13 & 0.6 & 22 & 1.0 & 22 & 1.0\end{array}$

$\begin{array}{lllllllllllll}\text { Medicamentos } & 2010 & 90.9 & 121 & 5.4 & 53 & 2.4 & 9 & 0.4 & 9 & 0.4 & 9 & 0.4\end{array}$

para razones no

médicas

$\begin{array}{lllllllllllll}\text { Otras drogas } & 2144 & 96.9 & 45 & 2.0 & 7 & 0.3 & 9 & 0.4 & 2 & 0.1 & 4 & 0.2\end{array}$

Fuente: elaboración propia $\mathrm{f}=$ frecuencia $\%=$ porcentaje $\mathrm{N}=2211$ 
Respecto al consumo de sustancias, se incluyeron preguntas relacionadas con la disponibilidad de las mismas para los estudiantes. Los resultados indican que los participantes consideran que el cigarro, es una de las sustancias más fáciles de conseguir (30.4\%), seguida del alcohol (27.1\%), la marihuana (19.2\%), los medicamentos para razones no médicas $(17.6 \%)$ y otras drogas $(11.6 \%)$.

En cuanto al riesgo percibido en el consumo regular de las tres principales sustancias (alcohol, cigarro y marihuana), encontramos que $14.6 \%$ de los participantes considera que consumir una o dos bebidas alcohólicas diariamente no representa riesgo para la salud, $12.9 \%$ no considera riesgo el fumar una o dos cajetillas diarias, mientras que $13.1 \%$ no considera un riesgo fumar diariamente marihuana.

\section{Diferencias en consumo de sustancias en cuanto a género y algunas variables escolares}

Al analizar por turno escolar, se encontraron diferencias significativas en el consumo de un vaso de alcohol en los últimos 30 días $(\mathrm{t}=-2.97, p=.003)$ encontrando medias superiores en los estudiantes del turno vespertino, en comparación con los del turno matutino. También se reportaron diferencias significativas en la variable del consumo de cinco vasos de alcohol $(\mathrm{t}=-2.072, p=.038)$, así como en el consumo de cigarro ( $\mathrm{t}=-2.663, p=.008)$, en ambos casos el turno vespertino obtuvo medias superiores que el matutino.

En la tabla cinco, se puede observar las diferencias entre el consumo de SPA y las variables grado académico y calificaciones. Se muestran las medias, valor $\mathrm{F} y$ significancia estadística. Encontrando diferencias significativas en todas las variables, a excepción del consumo de cigarro con el grado escolar (tabla 5). 
Tabla 5.

Consumo de SPA de acuerdo al grado escolar y las calificaciones del último reporte

\begin{tabular}{|c|c|c|c|c|c|}
\hline Consumo de sustancias & $\begin{array}{l}\text { Variable de } \\
\text { agrupación }\end{array}$ & Grupo & $\mathrm{M}(\mathrm{DE})$ & $\mathrm{F}$ & $p$ \\
\hline \multirow[t]{8}{*}{ Alcohol 1 bebida } & Grado & 1 & $1.39(.87)$ & 23.92 & .000 \\
\hline & & 2 & $1.61(1.0)$ & & \\
\hline & & 3 & $1.80(1.2)$ & & \\
\hline & Calificaciones & 6 & $1.63(1.1)$ & 8.14 & .000 \\
\hline & & 7 & $1.73(1.1)$ & & \\
\hline & & 8 & $1.65(1.1)$ & & \\
\hline & & 9 & $1.51(1.0)$ & & \\
\hline & & 10 & $1.24(.65)$ & & \\
\hline \multirow[t]{8}{*}{ Alcohol más de 5 bebidas } & Grado & 1 & $1.16(.61)$ & 13.59 & .000 \\
\hline & & 2 & $1.26(.75)$ & & \\
\hline & & 3 & $1.38(.88)$ & & \\
\hline & Calificaciones & 6 & $1.33(1.0)$ & 5.90 & .000 \\
\hline & & 7 & 1.32(.79) & & \\
\hline & & 8 & $1.32(.83)$ & & \\
\hline & & 9 & $1.20(.64)$ & & \\
\hline & & 10 & $1.09(.44)$ & & \\
\hline \multirow[t]{8}{*}{ Cigarros } & Grado & 1 & $1.22(.71)$ & 1.98 & .137 \\
\hline & & 2 & $1.29(.91)$ & & \\
\hline & & 3 & $1.31(.95)$ & & \\
\hline & Calificaciones & 6 & $1.48(1.0)$ & 8.36 & .000 \\
\hline & & 7 & $1.43(1.0)$ & & \\
\hline & & 8 & $1.31(.91)$ & & \\
\hline & & 9 & $1.18(.74)$ & & \\
\hline & & 10 & $1.07(.29)$ & & \\
\hline \multirow[t]{8}{*}{ Mariguana } & Grado & 1 & $1.14(.67)$ & 3.76 & .023 \\
\hline & & 2 & $1.23(.87)$ & & \\
\hline & & 3 & $1.26(.92)$ & & \\
\hline & Calificaciones & 6 & $1.24(.82)$ & 4.66 & .001 \\
\hline & & 7 & $1.29(1.0)$ & & \\
\hline & & 8 & $1.26(.90)$ & & \\
\hline & & 9 & $1.13(.65)$ & & \\
\hline & & 10 & $1.06(.46)$ & & \\
\hline
\end{tabular}

Fuente: elaboración propia

\section{Discusión}

Con respecto a las conductas violentas, encontramos que las peleas físicas son una de las conductas en las que más se involucran los estudiantes de secundaria. Cuatro de cada 10 adolescentes reportan haber participado en una pelea, por lo menos una vez al año, lo que genera el riesgo de consecuencias físicas, socioemocionales y económicas que afectan la salud (Pouwels \& Cillessen, 2013), este dato es trascendente, ya que a nivel mundial, está reportado que las heridas no intencionales son unas de las principales causas de mortalidad y discapacidad en jóvenes (Peyton et al., 2017), por otra parte, en el caso 
de México, para los varones jóvenes, la primera causa de muerte es la agresión (24.4\%), siendo la tercera $(9.5 \%)$ para las mujeres (Instituto Nacional de Estadística y Geografía, 2016); estos hallazgos, sugieren la necesidad de retomar estrategias de prevención, enfocadas hacia el tema de la violencia escolar, en específico aquella que implica agresiones físicas.

El bullying destaca en cuanto a violencia escolar, entre los resultados encontramos alrededor de $40 \%$ de los estudiantes que considera que el bullying es un problema importante dentro de su escuela. Aunado a lo anterior, se observaron diferencias entre el porcentaje de alumnos que considera que los adultos hacen cosas para detener el bullying y aquellos que opinan lo mismo sobre el resto de sus compañeros, siendo en este último apartado, donde se presenta un porcentaje más bajo. Este hallazgo concuerda con investigaciones que señalan que dentro del fenómeno del bullying, es común que los observadores hagan pocas cosas para detenerlo, cuando no mantienen una relación con la víctima, siendo en algunas ocasiones, sus principales promotores (Saarento \& Salmivalli, 2015). Lo anterior, sugiere la necesidad de reforzar aquellas estrategias de prevención en las escuelas, de manera que se promuevan intervenciones enfocadas a la inclusión y empoderamiento de los observadores dentro del proceso de prevención.

Por otra parte, en cuanto a la violencia comparada por género y calificaciones, se encontró que los varones y los estudiantes con más bajo desempeño académico, tienden a participar con mayor frecuencia en conductas violentas. Se encontraron diferencias en comportamientos como portar armas en la escuela, participar en peleas, ser amenazados con armas dentro de la escuela y pertenecer a pandillas. Estos resultados concuerdan con lo reportado en investigaciones que señalan la existencia de mayores conductas disruptivas y agresivas en los varones dentro de la escuela (Peyton, et al., 2017), además de considerar que el consumo de SPA y el bajo desempeño académico puede presentarse como una consecuencia de la conducta disruptiva o como un factor de riesgo para su generación (Lepore \& Kliewer, 2013). En todos los casos, los estudiantes del turno vespertino puntuaron con medias mayores que aquellos que asisten a la escuela por la mañana.

Los resultados evidencian que existe una diferencia en la percepción de la seguridad dentro de la escuela entre los estudiantes de ambos turnos. Una de las explicaciones podría ser que los alumnos del turno vespertino finalizan sus clases cuando anochece y está obscuro, volviéndose más vulnerables. Asimismo, llama la atención que los estudiantes del turno vespertino, obtuvieron mayores puntajes en la pertenencia a 
pandillas, al respecto, algunos autores señalan que la percepción de inseguridad en la escuela es uno de los factores motivantes para afiliarse a pandillas, buscando con ello protección (Forber et al., 2014).

En cuanto al consumo de sustancias, encontramos que el alcohol es la sustancia que los estudiantes refieren consumir con mayor frecuencia, seguida del cigarro y la marihuana, este resultado concuerda con lo reportado en distintas investigaciones (Aguilera, et al., 2007; Villatoro, et al., 2016). El porcentaje reportado en el consumo de otras sustancias psicoactivas es menor a $5 \%$ y debe ser tomado en cuenta para fines preventivos. Este hallazgo es relevante porque sugiere la existencia de un grado importante de experimentación e inclusive de abuso entre los estudiantes, el cual los pone en riesgo de distintos problemas de salud, entre los que se añaden el desarrollo de una adicción y la participación en situaciones de violencia (Vázquez, et al., 2014). Por lo tanto, es necesario retomar el tema en las escuelas secundarias, de manera que se recupere la información científica necesaria para desarrollar intervenciones que se conviertan en políticas públicas que impacten en la población.

Considerando que uno de los principales motivos para el inicio del consumo de alcohol y de tabaco es la disponibilidad, los resultados indican que para los adolescentes, el cigarro es el más fácil de conseguir, seguido del alcohol y la marihuana. Esto indica que existen espacios cercanos a la escuela, que facilitan el acceso de los estudiantes a este tipo de sustancias. En contraste, Trujillo, Pérez y Díaz-Granados (2011) señalan que el consumo de SPA, tiene una relación más significativa con la percepción de que los pares consumen, que con la propia disponibilidad de la sustancia, desatacando la influencia de los pares en el proceso de consumo. En la percepción de riesgo relacionado con el consumo de sustancias, encontramos que cerca del $15 \%$ no considera un riesgo el consumo regular de sustancias como alcohol, tabaco y marihuana. Esto nos habla de una aceptación social en el uso y abuso de sustancias, haciendo más complicado el proceso de autocuidado y prevención de adicciones (Salamó et al., 2010), destacando la importancia de retomar estrategias preventivas y educativas con los estudiantes.

Por otro lado, se realizaron comparaciones de la frecuencia de consumo reportada por los participantes. En cuanto al género, no se encontraron diferencias estadísticamente significativas, lo cual refleja los cambios observados en las últimas décadas, donde las mujeres alcanzaron la misma frecuencia de consumo que los hombres (Secretaria de Salud, 2016). Con respecto al turno, se encontraron diferencias en el consumo de alcohol, en cuanto a su uso (una bebida) y abuso (cinco bebidas), así como en el cigarro, 
observando medias superiores en los estudiantes del turno vespertino. Este resultado es relevante porque vuelve a evidenciar la existencia de diferencias importantes en aspectos conductuales entre los estudiantes, de acuerdo al turno al que asisten a la escuela, siendo un tema que requiere ser explorado con mayor profundidad en futuras investigaciones.

Las diferencias encontradas en cuanto al grado escolar estudiado y las calificaciones fueron similares, se observó que los estudiantes de grados superiores y con menor desempeño académico, presentaron puntajes superiores en el uso de alcohol (una bebida), abuso de alcohol (cinco bebidas) y uso de marihuana. Asimismo, los estudiantes con menor desempeño presentaron mayor consumo de cigarro que sus compañeros. Estos resultados coinciden con distintas investigaciones que señalan que el consumo de sustancias suele ir en aumento conforme el estudiante pasa de grado escolar, siendo un problema más frecuente en aquellos alumnos con bajo desempeño académico (Anderson \& Brown, 2011; Yamada, et al., 2016), situación que vuele más vulnerables a estas poblaciones.

Las principales limitaciones de este trabajo tienen que ver con el diseño y la muestra. Por un lado, es necesario incrementar el tamaño muestral e incluir estudiantes de otras localidades o estados, de manera que se aumente la representatividad de la misma a nivel nacional. Otra limitación es la relacionada a un posible sesgo en la respuesta sobre el consumo de drogas, debido a que los estudiantes podrían sentirse cohibidos al responder este tipo de preguntas.

Asimismo, se requiere realizar estudios longitudinales que permitan estudiar los fenómenos durante periodos más prolongados, consiguiendo establecer trayectorias y comparaciones. Por otra parte, sería importante incluir otros diseños metodológicos y otras variables provenientes del contexto familiar y social para poder comprender más integralmente los fenómenos estudiados.

No obstante las limitaciones detectadas, esta investigación mantiene implicaciones importantes para el desarrollo adolescente, al considerar la violencia escolar y el consumo de sustancias como factores asociados con la generación de consecuencias negativas. Con respecto a estos temas, es importante seguir realizando investigaciones que permitan recabar datos sobre las variables contextuales, familiares y escolares que promueven estos fenómenos. De esta manera, sería posible identificar e intervenir sobre los factores que promueven estas problemáticas, generando estrategias de prevención y promoción que favorezcan un bienestar y desarrollo positivo. 


\section{Referencias}

Aguilera, G. M. A., Muñoz, A. G. \& Orozco, M. A. (2007). Disciplina, violencia y consumo de sustancias nocivas en escuelas primarias y secundarias de México. Instituto Nacional de Evaluación Educativa.

https://www.inee.edu.mx/wp-content/uploads/2019/01/P1D230.pdf

Algozzine, B. \& Mcgee, J. (2011). Reported Occurrence and Perceptions of Violence in Middle and High Schools. The Clearing House, 84, 91-97. https://doi.org/10.1080/00098655.2010.524952

American Academy of Child and Adolescent Psychiatry. (2015). Los Adolescentes: el Alcohol y Otras Drogas.

https://www.aacap.org/AACAP/Families and Youth/Facts for Families/FF F-Spanish/Los-Adolescentes-el-Alcohol-y-Otras-Drogas-003.aspx

Anderson, K. \& Brown, S. (2011). Middle School Drinking: Who, Where, and When. Journal of Child \& Adolescent Substance Abuse, 20, 48-62. http://doi.org/10.1080/1067828X.2011.534362

Forber, A., Aragon, S. \& Espelage, D. (2014). The Influence of Gang Presence on Victimization in One Middle School Environment. Psychology of Violence, 4(1), 8-20. https://psycnet.apa.org/record/2013-11410-001

Bradshaw, C.P., Waasdorp, T.E., Debnam, K.J. \& Lindstrom, S. (2014). Measuring school climate in high school: a focus on safety, engagement, and the environment. Journal of School Health, 84 (1), 593-604.

https://onlinelibrary.wiley.com/doi/epdf/10.1111/josh.12186

Hutchinson, M. (2012). Exploring the impact of bullying on young bystanders. Educational Psychology in Practice, 28(4), 425-442. https://doi.org/10.1080/02667363.2012.727785

Iachini, A., Buettner, C., Anderson-Butcher, D. \& Reno, R. (2013). Exploring Students' Perceptions of Academic Disengagement and Reengagement in a Dropout Recovery Charter School Setting. Children \& schools, 35(2), 113120.

https://doi.org/10.1093/cs/cdt005

Instituto Nacional de Estadística y Geografía. (2016). Estadísticas a propósito del Día internacional de la juventud (15 a 29 años) 12 de agosto. http://www.inegi.org.mx/saladeprensa/aproposito/2016/juventud2016_0.pdf

Instituto Nacional de Psiquiatría Ramón de la Fuente Muñiz; Comisión Nacional Contra las Adicciones, Secretaría de Salud (2015). Encuesta Nacional de Consumo de Drogas en Estudiantes 2014: Reporte de Drogas. INPRFM.

http://www.conadic.salud.gob.mx/pdfs/investigacion/ENCODE ALCOHOL 2014.p $\underline{\mathrm{df}}$

Instituto Nacional de Psiquiatría Ramón de la Fuente Muñiz; Instituto Nacional de Salud Pública, Comisión Nacional Contra las Adicciones, Secretaría de Salud (2017). Encuesta Nacional de Consumo de Drogas, Alcohol y Tabaco 20162017: Reporte de Drogas. INPRFM.

http://inprf.gob.mx/psicosociales/archivos/ena/ENCODAT_DROGAS_20162017.pdf

Lepore, S. \& Kliewer W. (2013). Violence Exposure, Sleep Disturbance, and Poor Academic Performance in Middle School. J Abnorm Child Psychol, 41, p. 1179-1189. https://doi.org/10.1007/s10802-013-9709-0

Meneses, C., Markez, I., Romo, N., Uroz, J., Rua, A. \& Laespada, T. (2013). Diferencias de género en el consumo diario de tabaco e intensivo de alcohol 
en adolescentes latinoamericanos en tres áreas españolas (Andalucía, Madrid y País Vasco). Revista de la Asociación Española de Neuropsiquiatría, 33(119), 525-535.

http://scielo.isciii.es/pdf/neuropsiq/v33n119/original4.pdf

Milam, A., Furr-Holden, C. \& Leaf, P. (2010). Perceived School and Neighborhood Safety, Neighborhood Violence and Academic Achievement in Urban School Children. Urban Rev. 42, 458-467. https://doi.org/10.1007/s11256-010-0165$\underline{7}$

Organización Mundial de la Salud. (2004). La dependencia de sustancias es tratable, sostiene un informe de expertos en neurociencias. http://www.who.int/mediacentre/news/releases/2004/pr18/es/

Orozco, M.G. (2017). Factores sociodemográficos, familiares y escolares que influyen en la percepción del clima escolar en estudiantes de secundaria (Tesis de doctorado no publicada). Universidad de Guadalajara. Jalisco, México.

Peyton, R., Ranasinghe, S. \& Jacobsen, K. (2017). Injuries, Violence, and Bullying Among Middle School Students in Oman. Oman Medical Journal, 32(2), 98105. https://doi.org/10.5001/omj.2017.19

Pouwels, J. \& Cillessen, A. (2013). Correlates and Outcomes Associated with Aggression and Victimization Among Elementary-School Children in a LowIncome Urban Context. Journal of Youth \& Adolescence, 42(2), 190-205. https://doi.org/10.1007/s10964-012-9875-3

Román, M. \& Murillo, F. (2011). América Latina: violencia entre estudiantes y desempeño escolar. Revista CEPAL, 104, 37-54.

https://www.cepal.org/sites/default/files/gi/files/hoja_violencia_escolar_revistacepal _104.pdf

Saarento, S. \& Salmivalli, C. (2015). The Role of Classroom Peer Ecology and Bystanders' Responses in Bullying. Child Development Perspectives, 9(4), 201-205.

https://srcd.onlinelibrary.wiley.com/doi/pdf/10.1111/cdep.12140

Salamó, A., Gras, M. \& Font-Mayolas, S. (2010). Patrones de consumo de alcohol en la adolescencia. Psicothema, 22, (2), 189-195.

http://www.psicothema.com/pdf/3714.pdf

Secretaria de Salud (2016). Encuesta Nacional De Consumo De Drogas, Alcohol Y Tabaco 2016-2017: Resumen metodológico, 2016.

https://www.gob.mx/cms/uploads/attachment/file/234856/CONSUMO_DE_ DROGAS.pdf

Trujillo, A., Pérez, A. \& Díaz-Granados, O. (2011). Influencia de variables del entorno social sobre la ocurrencia de situaciones problemáticas asociadas al consumo de alcohol en adolescentes. Adicciones, 23(4), 349-356.

http://www.adicciones.es/index.php/adicciones/article/view/135/132

Valadez, J.A., Oropeza, R., Salazar, M.L. \& Martínez, K. (2018). La voz de los profesionales: componentes y sugerencias para los programas de prevención en adicciones. Revista Electrónica de Psicología Iztacala, 21(3), 796-816.

https://www.iztacala.unam.mx/carreras/psicologia/psiclin/vol21num3/Vol21No3Art1 . $\mathrm{pdf}$

Vázquez, M., Muñoz, M., Fierro, A., Alfaro, M., Rodríguez, M. \& Rodríguez, L. (2014). Consumo de sustancias adictivas en los adolescentes de 13 a 18 años y otras conductas de riesgo relacionadas. Rev Pediatr Aten Primaria, 16, 125134. 
https://www.redalyc.org/pdf/3666/366634304005.pdf

Villatoro, J., Medina-Mora, M.E., Martín del Campo, R., Fregoso, D., Bustos, M.C., Resendiz E., ... Cañas, V. (2016). El consumo de drogas en estudiantes de México: tendencias y magnitud del problema. Salud mental, 39 (4), 193-203.

https://doi.org/10.17711/SM.0185-3325.2016.023

Yamada, S., Pepler, D., Jiang, D., Cappadocia, M., Craig, W. \& Connolly, J. (2016). Developmental Trajectories of Adolescent Substance Use. Journal of Child \& Adolescent Substance Abuse, 25(1), 33-48. http://doi.org/10.1080/1067828X.2014.884482

Yarnell, L., Pasch, K., Brown, S., Perry, C. \& Komro, K. (2014). Cross-Gender Social Normative Effects for Violence in Middle School: Do Girls Carry a Social Multiplier Effect for At-Risk Boys? Journal Youth Adolescence, 43, 1465-1485.

https://link.springer.com/article/10.1007/s10964-014-0104-0 